\title{
Westernization of Lifestyle Causes Obesity-Associated Metabolic Disorders in the Japanese Population
}

\author{
Masayasu Yoneda*, Haruya Ohno and Kenji Oki
}

Department of Molecular and Internal Medicine, Institute of Biomedical \& H ealth S ciences, Hiroshima University, 1 -2-3 Kasumi, Minami-ku, Hiroshima 7348551, Japan

*Corresponding author: Masayasu Yoneda, Department of Molecular and Internal Medicine, Institute of Biomedical \& Health Sciences, Hiroshima University, 1-2-3 Kasumi, Minami-ku, Hiroshima 7348551, Japan, Tel: 81822575198; E-mail: masayone17@hiroshima-u.ac.jp

Received date: Oct 10, 2016; Accepted date: Oct 16, 2016; Published date: Oct 21, 2016

Citation: Yoneda M, Ohno H, Oki K (2016) Westernization of Lifestyle Causes Obesity-Associated Metabolic Disorders in the Japanese Population. J Obes Eat Disord 2:2. doi: 10.4172/2471-8203.100024

Copyright: (c) 2016 Yoneda M, et al. This is an open-access article distributed under the terms of the Creative Commons Attribution License, which permits unrestricted use, distribution, and reproduction in any medium, provided the original author and source are credited.

\section{Abstract}

In recent years, the percentage of obese individuals has been rising in many countries, accompanied by a sharp increase in the prevalence of obesity-associated metabolic disease, such as metabolic syndrome and diabetes mellitus. We recently compared the medical survey data of each generation between two Japanese populations with different lifestyles, i.e., the group of native Japanese living in Hiroshima, Japan, and the group of Japanese-Americans (Japanese people who migrated to Hawaii or Los Angeles, USA, and their offspring), focusing on differences in nutrient intake and the prevalence of obesity, metabolic syndrome, and diabetes mellitus. Japanese-Americans tended to consume a high-fat and high-simple carbohydrate diet, and had a higher prevalence of obesity-associated metabolic disease than native Japanese living in Japan. However, the detailed comparison among Japanese-Americans revealed that experience with the Japanese lifestyle during childhood suppressed the onset of obesity in later years.

Keywords: Westernization of lifestyle; Metabolic syndrome; Obesity; Diabetes mellitus; Japanese-Americans

\section{Introduction}

Overseas immigration of the Japanese people began in the latter half of the 19th century. In those days, lifestyle related to diet and exercise differed largely between Japan and the USA. The lifestyle of first-generation Japanese-Americans (JA-1) who were born in Japan and immigrated to the USA underwent rapid westernization (change from the Japanese to the American style) after immigration, and their offspring born and raised in the USA (second- or later-generation: JA-2) were exposed to the American lifestyle since birth.

Since 1970, we have been conducting an epidemiological study titled "the Hawaii-Los Angeles-Hiroshima study," which involves two populations of Japanese people without any differences in genetic predisposition but who lives under different environmental factors: the group of JapaneseAmericans having acquired an American lifestyle and the group of native Japanese living in Hiroshima following the Japanese lifestyle. By comparing the medical survey data between these two groups, we previously reported the influence of lifestyle westernization on the features of illness among Japanese people.

Using the data so far collected in the Hawaii-Los AngelesHiroshima study, this paper will describe the influence of the Japanese or American lifestyle on obesity-associated metabolic disorders in Japanese people through comparison of the group of native Japanese living in Japan and the group of JapaneseAmericans, and via analysis of each generation (JA-1 and JA-2) of Japanese-Americans.

\section{The Hawaii-Los Angeles-Hiroshima Study}

We started our medical research on Japanese-Americans in Hilo and Kona, on Island of Hawaii, in 1970, and in Los Angeles, California, in 1978. Medical examinations have been conducted every few years in each district. By 2015, we performed these medical examinations a total of 24 times, and the total number of study participants has exceeded 13,000 (Figure 1).

All subjects provided written informed consent to participate in the examinations. This study was approved by the ethics committee of Hiroshima University and the Hiroshima Kenjin-kai Associations of Island of Hawaii and Southern California.

In the morning, following an overnight fast, each subject underwent an interview, physical examination, blood pressure measurements, and venous blood sampling. An oral glucose tolerance test was performed on the subjects without diabetes mellitus. Each blood sample was centrifuged, and the obtained serum samples were immediately frozen and stored until analysis. 


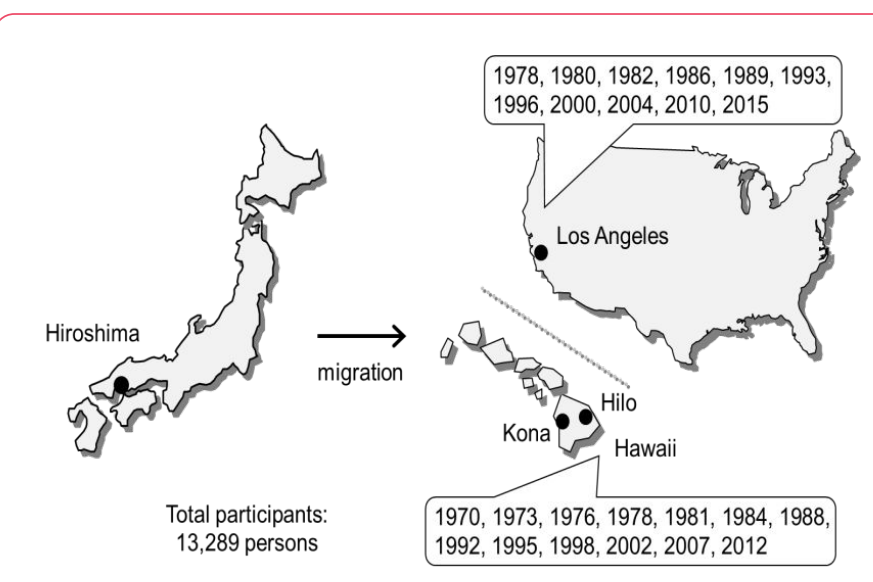

Figure 1: The Hawaii-Los Angeles-Hiroshima study.

\section{Energy Intake among Native Japanese and Japanese-Americans}

According to the survey data from 1978 to 1988 , the total energy intake differed little between Japanese-Americans and native Japanese, whereas the percentage of nutrients intake differed between the two groups (higher percentage of fat intake in the male and female Japanese-American group). This resulted in a higher animal fat, higher simple carbohydrate, and lower complex carbohydrate dietary style in the male and female Japanese-American group than in the Japanese group [1].

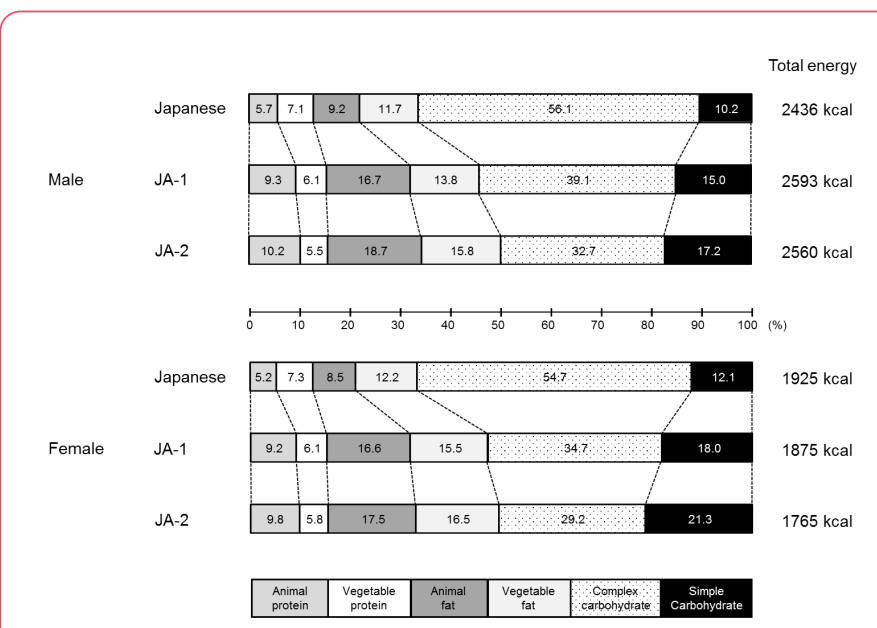

Figure 2: Nutrient ingestion in different age groups.

Survey in 1992 to 1995.594 native Japanese (273 male and 321 female); 477 first-generation Japanese-Americans (JA-1; 181 male and 296 female); 549 second- or later-generation Japanese-Americans (JA-2; 164 male and 385 female).

From the survey data conducted from 1992 to 1995, we divided the Japanese-Americans into two subgroups (JA-1 and JA-2) for comparison of nutritional data (Figure 2) [2].

Among male individuals, the total energy intake differed little between the Japanese group, JA-1 group, and JA- 2 group. On the other hand, among female individuals, total energy intake was lower in the JA-1 group and even lower in the JA-2 group than in the Japanese group. When analyzing the percentage of nutrient intake, we found that the percentage of animal protein, animal/ vegetable fat, and simple carbohydrate intake was highest in the JA-2 group, followed by the JA-1 group and the Japanese group, regardless of sex; the percentage of complex carbohydrate intake was highest in the Japanese group, followed by the JA-1 group and the JA-2 group.

\section{Prevalence of Obesity-Associated Metabolic Disease among Native Japanese and Japanese-Americans}

We compared the prevalence of diabetes mellitus at age 40 and older between the Japanese-American groups and the Japanese group using the survey data from 1978 to 1988. Interestingly, the prevalence of diabetes was two to three times higher in the Japanese-Americans of each age group living in Hawaii and Los Angeles, after adjustment for age and sex, as compared with the Japanese group [3].

The prevalence of metabolic syndrome was analyzed separately for male and female individuals aged 30 and older using the survey data in 2004 [4]. In both of the analyses using the International Diabetes Federation (IDF) criteria made public in 2005 and the American Heart Association/National Heart, Lung and Blood Institute (AHA/NHLBI) criteria for diagnosis of metabolic syndrome, the prevalence of metabolic syndrome among male individuals was significantly higher in the JapaneseAmerican group than in the Japanese group. The prevalence of metabolic syndrome among female individuals was significantly higher in the Japanese-American group than in the Japanese group $(27.7 \%$ vs. $14.4 \%$ ) when the $\mathrm{AHA} / \mathrm{NHLBI}$ criteria were used, whereas it did not differ significantly between the Japanese group (6.3\%) and the Japanese-American group (4.5\%) when metabolic syndrome was diagnosed using the IDF criteria, for which abdominal obesity is an indispensable diagnostic element.

The JA-2 group practiced "Kibei" (assignment of the USA-born infants and small children to relatives in Japan, with return to the USA after receiving education in Japan). Hankin et al. conducted a nutritional survey of 6,663 male JapaneseAmericans aged 45 to 69 living in Oahu, Hawaii. They reported that JA-2 individuals who experienced Kibei (i.e., having lived in Japan) more frequently ingested Japanese-style foods (rice, tofu, miso soup, green tea, etc.) and less frequently ingested Western-style foods (bread, meat, ham, sausage, coffee, etc.) than JA-2 individuals without experience of Kibei [5]. Furthermore, Huang et al. reported that among the 7,959 male Japanese-Americans living in Oahu, physical activity level was higher and percentage of fat ingestion lower as the duration of living in Japan increased [6].

In our survey from 2007 to 2010, Kibei was defined as the history of staying in Japan for $\geq 5$ years before the age of 18 . The prevalence of obesity and diabetes among Japanese-Americans 
aged 30 and older was analyzed in relation to generation and experience with Kibei (Figure 3) [7].
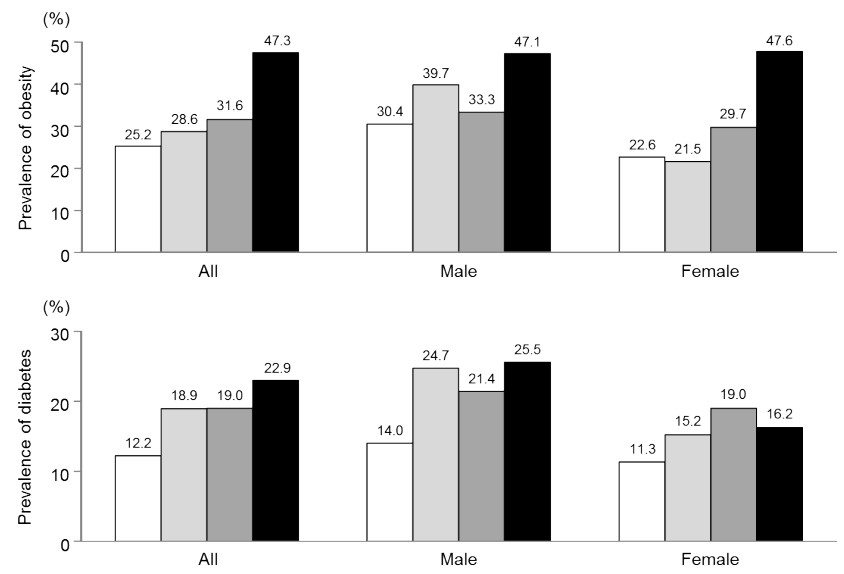

Figure 3: Percentage of obese individuals and prevalence of diabetes by generation and Kibei experience.

Survey in 2007 to 2010.. : 516native Japanese (171 male and 345 female); :444first-generation Japanese-Americans (JA-1; 174 male and 270 female); : 79 second-orlater-generation Japanese-Americans who experienced Kibei (Kibei-positive JA-2 group; 42 male and 37 female); $: 258$ second-or latergeneration Japanese-Americans who did not experience Kibei (non-Kibei JA-2 group; 153 male and 105 female).

In that survey, the percentage of obese individuals (body mass index $\geq 25$ ) differed little between the Japanese group, JA-1 group, and Kibei-positive JA-2 group, whereas it was significantly higher in the non-Kibei JA-2 group (percentage of obese individuals close to $50 \%$ ). On the other hand, the prevalence of diabetes was higher in all Japanese-American groups than in the Japanese group, but there was no significant difference in this parameter between the JA-1, Kibei-positive JA-2, and non-Kibei JA-2 groups.

In brief, these results indicate that Japanese people continuously exposed to the American lifestyle for a long period after birth were more likely to become obese. Onset of obesity was suppressed in Japanese people who lived in Japan during childhood (e.g., JA-1 and Kibei-positive JA-2), although onset of diabetes was not suppressed in these groups (Figure 4). Japanese-Americans are known to be genetically predisposed to low insulin secretion. The results suggest that abnormal glucose tolerance is likely to develop before the onset of obesity if Japanese people follow the American lifestyle even for a relatively short period of time.

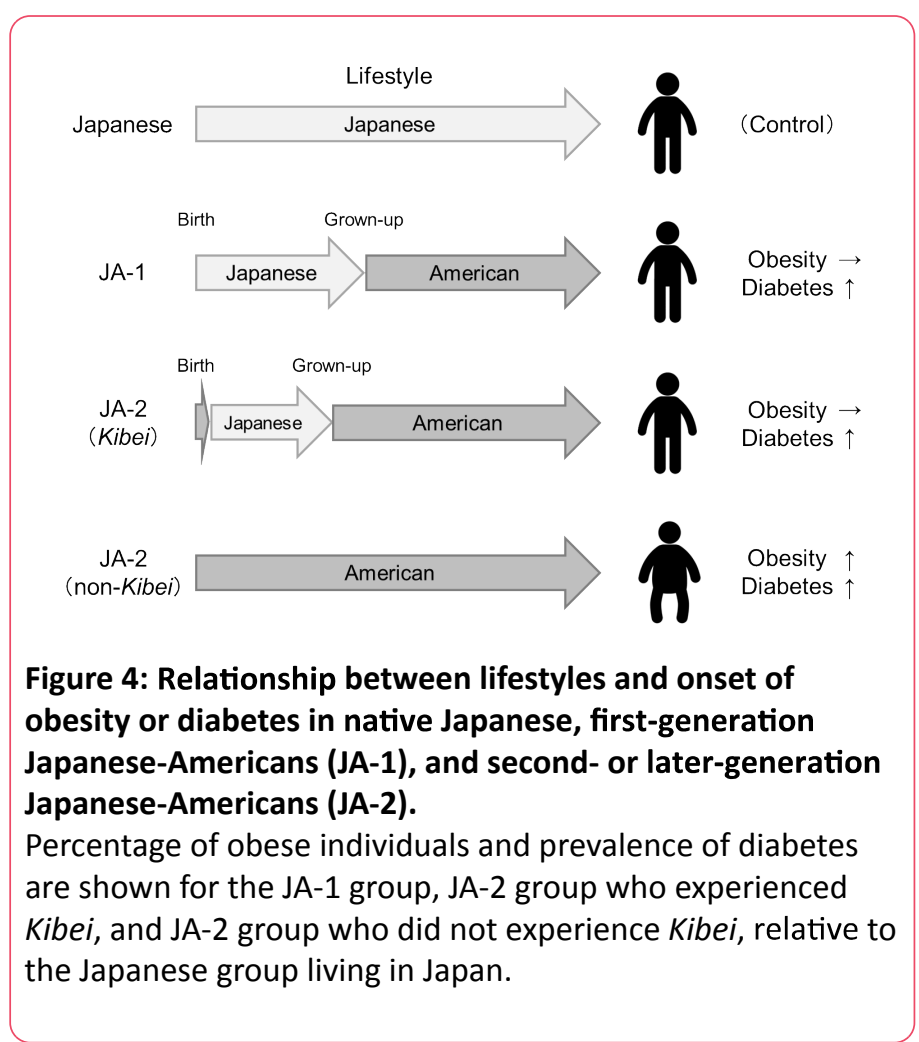

\section{Conclusion}

On the basis of this comparison of survey data between native Japanese and Japanese-Americans (two groups identical in genetic predisposition but differing in lifestyle from each other), we believe definitely that obesity-associated metabolic disorders can arise from westernization of the lifestyle of Japanese people. The results of the Hawaii-Los Angeles-Hiroshima study indicate the importance of acquiring a Japanese dietary style beginning in childhood so that onset of obesity-associated metabolic disease may be prevented.

\section{Acknowledgments}

This survey was supported by the members of the Hiroshima Kenjin-kai Associations of Island of Hawaii and Southern California.

\section{Reference}

1. Egusa G, Murakami F, Ito C, Matsumoto $Y$, Kado S, et al. (1993) Westernized food habits and concentrations of serum lipids in the Japanese. Atherosclerosis 100: 249-255.

2. Egusa G, Watanabe H, Ohshita K, Fujikawa R, Yamane K, et al. (2002) Influence of the Extent of Westernization of Lifestyle on the Progression of Preclinical Atherosclerosis in Japanese Subjects. J Atheroscler Thromb 9: 299-304.

3. Hara H, Egusa G, Yamakido M, Kawate R (1994) The high prevalence of diabetes mellitus and hyperinsulinemia among the Japanese-Americans living in Hawaii and Los Angeles. Diabetes Res Clin Pract 24: S37-S42. 
4. Yoneda M, Yamane K, Jitsuiki K, Nakanishi S, Kamei N, et al. (2008) Prevalence of metabolic syndrome compared between native Japanese and Japanese-Americans. Diabetes Res Clin Pract 79: 518-522.

5. Hankin JH, Nomura A, Rhoads GG (1975) Dietary patterns among men of Japanese ancestry in Hawaii. Cancer Res 35 : 3259-3264.

6. Huang B, Rodriguez BL, Burchfiel CM, Chyou PH, Curb JD, et al. (1996) Acculturation and prevalence of diabetes among
Japanese-American men in Hawaii. Am J Epidemiol 144: 674-681.

7. Shiwa M, Yoneda M, Nakanishi S, Oki K, Yamane K, et al. (2015) Japanese Lifestyle during Childhood Prevents the Future Development of Obesity among JapaneseAmericans. PLoS One 10: e0120804. 